\title{
Editorial
}

\section{'Yaba' Addiction-A Rising Concern in Bangladesh}

SA Fattah

Yaba, a Thai word for 'crazy medicine' is a recently emerged substance of abuse in Southeast and East Asia including Bangladesh. Speedy rise of its popularity and use in younger generation, particularly in affluent circles, is a great concern for the parents and the society. It is estimated that there are about 4.6 million regular users of yaba in Bangladesh and the number is increasing alarmingly everyday ${ }^{1}$.

Yaba is the tablet form of the synthetic drug 'methamphetamine' often mixed with 'caffeine' and/or 'heroin' in varying proportion. Originally used as nasal decongestant and anti-asthmatic, it was used by Hitler for his soldiers in World War-II to combat fatiguability and increase endurance ${ }^{2}$. Later it became popular as a hallucinogenic and stimulant club drug in Thailand, Myanmar and adjacent regions. Yaba was introduced to Bangladesh from Myanmar in 1999 and spreaded rapidly to Dhaka and all over the country. Now it is the number one ecstasy drug replacing heroin or phensydil due to its easy transportability and strong addictive potential.

Methamphetamine can be ingested, inhaled, snorted or injected intravenously. Commonly, it is available as small rounded pills of different colour e.g. red, orange or green. In all forms it is dangerous and has a high potential for addiction and physical harm. Moderate to chronic use of yaba may lead to physical and psychological dependence, even death. Those who coming off the drug are also at high risk of withdrawal symptoms including depression and suicidal tendency ${ }^{2}$.

Yaba exerts its stimulant effects by directly stimulating central nervous system and also by stimulating sympathetic nervous system via releasing catecholamines and inhibiting its uptake ${ }^{3}$. Its action starts 5-6 minutes after ingestion and lasts for hours, even longer with higher dosages ${ }^{3}$. Its effects depend on the route of use and dose. Effects include euphoria, increased alertness, wakefulness, irritability, aggression, decreased appetite, hot flushes, dry mouth etc. Chronic use results in tremor, hypertension, hallucination, damage to small blood vessels of brain and heart, psychotic episodes, paranoid delusion, violent behavior, hyperthermia, convulsion, agitation, anxiety, nervousness and psychosis similar to schizophrenia. Yaba has the property of development of tolerance leading to progressive increase in amount of drug requirement ${ }^{3}$. A regular user needs 8-10 tablets daily on average and each tablets costs about Taka 6001000. There is no standard diagnostic test and treatment protocol yet. History of drug intake, clinical features supported sometimes by detection of it and its metabolite in urine is the method of diagnosis. No pharmacological agent is satisfactory for treatment and prevention of addiction. Behavioral therapy for understanding and motivation is the mainstay of management ${ }^{4}$.

The social effect of drug abuse is also enormous. It is reported that approximately 70 percent of crimes including theft, robbery, kidnapping, murder are directly or indirectly related to drugs ${ }^{1}$. Bangladesh is not a major illicit drug producing country but its critical location in the middle of two of the world's largest narcotics producing belts - the Golden Triangle (Myanmar, Thailand, Vietnam and Laos) and the Golden Crescent (Afghanistan, Iran and Pakistan) makes it vulnerable to drug trade. In recent years, large amount of illicit drugs are also produced in India. Myanmar is the largest producer of yaba globally and via Teknaf and Cox's Bazar it is smuggled to Bangladesh $^{1}$. According to an intelligence report, at least 13 syndicates are active in the borders, 131 syndicates across the country and more than 100000 people are engaged in this ruthless business ${ }^{1}$. This situation indicates how difficult it is to eliminate this dangerous evil from the society.

Drug trafficking and abuse is a multifactorial problem and a holistic approach is needed to address this. Strict role of law-enforcing authority, diplomatic cooperation with neighboring countries, poverty alleviation, control of corruption - all are important aspects. But the most important step to begin with is awareness building and motivation of people of all sphere of life. Parents should be more conscious about caring their children. This combined approach is the only key to win the fight against this dreadful evil of yaba and other illicit drug abuse in the society.

\section{References :}

1. Preetha SS. Chasing the Dragon. In: The Daily Star Weekend Magazine. Volume 11, Issue 22, June 01, 2012.

2. Olson KR. Poisoning. In: Stephene J. McPhee, Maxine A. Papadakis, editors. Current Medical Diagnosis and Treatment, 49th ed. McGrawHill Lange; 2010. p.1430.

3. Tomb DA. Psychiatry, 5th ed. Williams and Wilkins; 1995.p.166-68.

Dr. Sk. Abdul Fattah DTCD, FCPS (Med) Associate Professor of Medicine Faridpur Medical College, Faridpur. E-mail: skfattah@gmail.com 